\title{
On some classes of linear spaces
}

by

S. MAZUR (Warszawa) and W. ORLICZ (Poznań)

Since the fundamental papers of F. Riesz ([5], [6]) the spaces $L^{a}$ and $l^{\alpha}$ are reckoned among the classical examples of linear normed spaces in the functional analysis. The space $l^{\alpha}$, where $\alpha>0$, is the space of sequences $\left\{t_{n}\right\}$ such that the series $\sum_{v}^{\infty}\left|t_{v}\right|^{a}$ converges; $L^{a}$, where $\alpha>0$, denotes the space of measurable functions in $(a, b)$ for which the integral

$$
\int_{a}^{b}|x(t)|^{\alpha} d t
$$

is finite. In the period between the wars and after World War II there appeared several papers dealing with generalizations of the spaces $l^{\alpha}$ and $L^{a}$. The idea of these generalizations is based upon the following. Let $N$ be a non-negative function defined for all real values. One considers the class $X^{N}$ of sequences for which the series

$$
\varrho(x)=\sum_{\nu=1}^{\infty} N\left(t_{\nu}\right), \quad x=\left\{t_{n}\right\},
$$

converges. It may be proved under very general and natural supplementary conditions about the function $N$ that $X^{N}$ are Banach spaces. In the case $N(u)=|u|^{\alpha}$, where $\alpha \geqslant 1$, these conditions are satisfied, and $l^{\alpha}$ form a particular case of the spaces $X^{N}$. An analogous situation holds for the spaces of measurable functions in $(a, b)$. Denoting by $X^{N}$ the class of measurable functions in $(a, b)$ for which the integral

$$
\varrho(x)=\int_{a}^{b} N(x(t)) d t
$$

exists and is finite, we may prove under certain hypotheses on $N$ that $X^{N}$ is a Banach space. The spaces $L^{a}$ with $\alpha \geqslant 1$ are a particular case of the spaces $X^{N}$ corresponding to $N(u)=|u|^{a}$.

Studia Mathematica T. XVII 
In this paper we are concerned with the examination of the spaces $X^{N}$ of sequences and those of functions from a very general standpoint. Under very slim hypotheses about the function $N$ we deal with the following problems: a) in which case are the spaces $X^{N}$ linear? b) which are the necessary and sufficient conditions for the function $N$ in order to make it possible in the linear space $X^{N}$ to define an $F$ - or $B$-norm such that the relation $\left\|x_{n}\right\| \rightarrow 0$ as $n \rightarrow \infty$ be equivalent to $\varrho\left(x_{n}\right) \rightarrow 0$ as $n \rightarrow \infty$ ?

The main results of the paper are contained in theorems 3 , 6 , from which it follows that the well known sufficient conditions on $N$ asserting that $X^{N}$ is a $B$-space are in some sense necessary.

The paper consists of two paragraphs. In the first we deal with the spaces of sequences for which the obtained results have a more complete character than for the spaces of functions considered in the second paragraph.

Throughout this paper $N, M, \ldots$ denote non-negative functions defined for all real values; in $\S 1, x, y, \ldots$ denote sequences $\left\{t_{n}\right\},\left\{s_{n}\right\}, \ldots$ with real terms, in $\S 2, x(t), y(t), \ldots$ stand for real measurable functions in $(0,1)$. The spaces of sequences $X^{N}$ and of measurable functions are always understood to be linear under the usual definitions of addition and multiplication by scalars. Equality of the measurable functions $x$ and $y$ means that $x(t)=y(t)$ for almost every $t$.

1.1. Given a function $N$, we shall write in this section

$$
\varrho_{N}(x)=\sum_{\nu=1}^{\infty} N\left(t_{p}\right)
$$

We shall also write $\varrho(x)$ instead of $\varrho_{N}(x)$ if the omission of the subscript will not cause misunderstanding about the involved function. $N$. By $X^{N}$ we shall denote the set of all sequences for which $\varrho_{N}(x)<\infty$. We shall frequently suppose that $N$ satisfies the following condition:

(*) $\quad N\left(t_{n}\right) \rightarrow 0$ as $n \rightarrow \infty$ if and only if $t_{n} \rightarrow 0$ as $n \rightarrow \infty$.

The condition $(*)$ implies in particular $N(0)=0$ and $N(t) \neq 0$ for $t \neq 0$.

The functions $N$ and $M$ will be called equivalent, in symbols $N \sim M$, if the following property is satisfied:

There are constants $A>0, B>0, \varepsilon>0$ such that $N(t) \leqslant B M(t)$ for $M(t) \leqslant \varepsilon, M(t) \leqslant A . N(t)$ for $N(t) \leqslant \varepsilon$.

It is easily seen from this definition that

1.12. For $N$ and $M$ satisfying the condition (*), $N \sim M$ if and only if simultaneously $N(t) \leqslant B M(t)$ and $M(t) \leqslant A N(t)$ in some neighbourhood of 0 .
In the sequel we shall need the following lemmata:

1.13. Let $M(t, s)$ and $N(t, s)$ be non-negative functions defined for all real values of $t$ and $s$. A necessary and sufficient condition that the convergence of the series $\sum_{v=1}^{\infty} M\left(t_{v}, s_{v}\right)$ imply the convergence of the series $\sum_{v=1}^{\infty} N\left(t_{v}, s_{v}\right)$ is the existence of two positive constants. $C$ and $\varepsilon$ such that

$$
N(t, s) \leqslant C M(t, s) \quad \text { when } \quad M(t, s)<\varepsilon .
$$

Sufficiency being obvious, we prove only the necessity. First it is easily seen that $M(t, s)=0$ implies $N(t, s)=0$. Suppose $(+)$ is not satisfied, then there must exist $t_{n}, s_{n}$ such that $N\left(t_{n}, s_{n}\right) \geqslant n M\left(t_{n}, s_{n}\right)$ and $M\left(t_{n}, s_{n}\right) \leqslant 1 / n^{2}$ for $n=1,2, \ldots$ We may suppose that $M\left(t_{n}, s_{n}\right) \neq 0$. Let us choose positive integers $p_{n}$ such that $1 / n^{2} \leqslant p_{n} M\left(t_{n}, s_{n}\right) \leqslant 2 / n^{2}$ for $n=1,2, \ldots$ Let $t_{n}^{\prime}=t_{r}, s_{n}^{\prime}=s_{r}$ for $p_{0}+\ldots+p_{r_{-1}}<n \leqslant p_{0}+\ldots+p_{r}$ as $r=1,2, \ldots$ (we set $p_{0}=0$ ). Since

$$
\sum_{\nu=1}^{\infty} M\left(t_{\nu}^{\prime}, s_{\nu}^{\prime}\right)<\infty, \quad \sum_{p=1}^{\infty} N\left(t_{\nu}^{\prime}, s_{\nu}^{\prime}\right)=\infty
$$

we are led to a contradiction.

Setting $M(t, s)=M(t), N(t, s)=N(t)$ for arbitrary $t, s$ we find from 1.13 that

1.14. A necessary and sufficient condition that $\varrho_{M}(x)<\infty$ imply $\varrho_{N}(x)<\infty$ is the existence of two constants $B>0, \varepsilon>0$ such that $N(t) \leqslant B M(t)$ for $M(t)<\varepsilon$.

1.2. Let $\varphi_{n}(\omega)$ denote for $n=1,2, \ldots$ non-negative measurable functions defined in $(-\delta, \delta)$, satisfying for a certain constant $K>0$ the inequality

$$
\varphi_{n}\left(\omega_{1}+\omega_{2}\right) \leqslant K\left[\varphi_{n}\left(\omega_{1}\right)+\varphi_{n}\left(\omega_{2}\right)\right] \quad \text { for } \quad \omega_{1}, \omega_{2} \epsilon E,
$$

$E$ being a measurable set in $(-\delta, \delta)$ such that $|E|>\frac{7}{4} \delta$.

Then

A. If $\varphi_{n}(\omega) \rightarrow 0$ as $n \rightarrow \infty$ for $|\omega|<\delta$, then for each $\varepsilon>0$ the following inequality is satisfied for almost all n's:

$$
\varphi_{n}(\omega) \leqslant \varepsilon \quad \text { for } \quad|\omega|<\delta / 4 .
$$

B. If the sequence $\varphi_{n}(\omega)$ is bounded for each $|\omega|<\delta$, then there is a constant $L>0$ such that

$$
\varphi_{n}(\omega) \leqslant L \quad \text { for } \quad|\omega|<\delta / 4 \text { and } n=1,2, \ldots
$$

Ad A. Given $\varepsilon>0$ let us set for $k=1,2, \ldots$ 


$$
\begin{aligned}
& T_{k}^{+}=\left\{\omega: \varphi_{n}(\omega) \leqslant \varepsilon \text { for } n \geqslant k, \omega \in E \cap(0, \delta)\right\}, \\
& T_{k}^{*}=\left\{\omega: \varphi_{n}(\omega) \leqslant \varepsilon \text { for } n \geqslant k, \omega \in E \cap(-\delta, 0)\right\} .
\end{aligned}
$$

Let us denote by $T_{k}^{-}$the set symmetrical to $T_{k}^{+-}$with respect to the point 0. Since $\lim T_{k}^{+}=(0, \delta) \cap E, \lim T_{k}^{*}=(-\delta, 0) \cap E$ we infer that $\lim _{k}\left|T_{k}^{*}\right|=|(0, \delta) \cap E|, \lim _{k}\left|T_{k}^{-}\right|=|(-\delta, 0) \cap E|, \lim _{k}\left|T_{k}^{*}\right|=|(-\delta, 0) \cap E|$. It follows that it is possible to choose $k_{0}$ so large that for the set $S^{-}=T_{k_{0}}^{*} \cap T_{k_{0}}^{-}$and for the set $S^{+}$symmetrical to it (with respect to 0 ) the inequalities $\left|S^{-}\right|>\frac{3}{4} \delta,\left|S^{+}\right|>\frac{3}{4} \delta$ respectively are satisfied. Every translation $S_{\omega}^{+}$of the set $S^{+}$by the length w such that $|\omega| \mid \leqslant \delta / 4$ has common points with $S^{+}$. It follows that

$(++) \quad \omega=\omega_{1}-\omega_{2} \quad$ where $\quad \omega_{1} \epsilon S^{++} \subset T_{k_{0}}^{++},-\omega_{2} \in S^{--} \subset T_{k_{0}}^{*}$ if $|\omega|<\delta / 4$

By $(+)$ we have $\varphi_{n}(\omega) \leqslant K\left[\varphi_{n}\left(\omega_{1}\right)+\varphi_{n}\left(-\omega_{2}\right)\right] \leqslant K \cdot 2 \varepsilon$ for $|\omega|<\delta / 4$ and $n \geqslant k_{0}$.

$A d$ B. Let us set for $k=1,2, \ldots$

$$
\begin{aligned}
& T_{k}^{+}=\left\{\omega: \varphi_{n}(\omega) \leqslant k \text { for } n \geqslant 1, \omega \in E \cap(0, \delta)\right\}, \\
& T_{k}^{*}=\left\{\omega: \varphi_{n}(\omega) \leqslant k \text { for } n \geqslant 1, \omega \in E \cap(-\delta, 0)\right\} .
\end{aligned}
$$

Starting with these sets let us define the sets $S^{+}$and $S^{-}$as above. Then $(++)$ remains true, whence by $(+)$ it follows

$\left(\begin{array}{l}++ \\ +\end{array}\right) \varphi_{n}(\omega) \leqslant K\left[\varphi_{n}\left(\omega_{1}\right)+\varphi_{n}\left(-\omega_{2}\right)\right] \leqslant K \cdot 2 k_{0} \quad$ for $\quad|\omega| \leqslant \delta / 4, n \geqslant 1$.

1.3. (a) We have $X^{N}=X^{\text {is }}$ if and only if $N \sim M$;

(b) The space $X^{N}$ is identical with the space of all sequences if and only if $N(t)=0$ for all $t$.

(c) The space $X^{N}$ is identical with the space of the sequences $\left\{t_{n}\right\}$ for which $t_{n}=0$ for almost all $n$ if and only if $N(0)=0$ and there is $\delta>0$ such that $N(t)>\delta$ for $t \neq 0$.

$A d$ (a). It follows immediately by 1.14 and by the definition of equivalence.

$A d$ (b). Sufficiency being trivial, let us suppose that $N(t) \neq 0$; then for the sequence $x=(t, t, \ldots)$ we have $\varrho_{N}(x)=\infty$, whence $X^{N}$ could not contain all the sequences.

$A d$ (c). The sufficiency is trivial. Now let $X^{N}$ consist only of sequences whose almost all elements are equal to zero: then the sequence $(0,0, \ldots)$ is in $X^{N}$, whence $N(0)=0$. Let us now suppose that $N\left(t_{n}\right) \rightarrow 0$ where $t_{n} \neq 0$. Then for a subsequence of $t_{n}$ we have $\varrho_{N}(x)<\infty$ and $X^{N}$ contains a sequence with infinitely many terms different from 0 .

1.4. The following conditions are necessary and sufficient that the space $\bar{X}^{N}$ be linear: (a) There are constants $C>0, \varepsilon>0$ such that

$$
N(t+s) \leqslant C[N(t)+N(s)] \quad \text { for } \quad N(t) \leqslant \varepsilon, N(s) \leqslant \varepsilon ;
$$

(b) For each $\omega$ there are constants $D_{\omega}>0, \varepsilon_{\omega}>0$ such that

(c) $N(0)=0$.

$$
N(\omega t) \leqslant D_{\omega} N^{\prime}(t) \quad \text { for } \quad N(t)<\varepsilon_{\omega}
$$

To prove (a) let us set $M(t, s)=N(t)+N(s), N(t, s)=N(t+s)$. Since $\varrho_{N}(x)<\infty, \varrho_{N}(y)<\infty$ implies $\varrho_{N}(x+y)<\infty$, it is sufficient to apply 1.13 to $M(t, s)$ and $N(t, s)$. To prove (b) we apply 1.14 to the functions $N(\omega t)$ and $N(t)$ considering that from $\varrho_{N}(x)<\infty$ follows $\varrho_{N}(\omega x)<\infty$. (c) follows from the trivial remark that $X^{N}$ contains the sequence $(0,0, \ldots)$ if and only if $N(0)=0$.

1.5. Let the function $N$ be measurable and let the space $X^{N}$ be linear. Then one of the following three cases holds:

(a) $\quad N(t)=0$ for every $t$;

(b) $\quad N(0)=0$, there is a $\delta>0$ such that $N(t) \geqslant \delta$ as $t \neq 0$;

(c) $\quad N$ satisfies the condition (*).

Let us suppose that the cases (a) and (b) are not satisfied. Suppose that for $t_{n}$ we have $\left|t_{n}\right| \geqslant \varrho>0$ and $N\left(t_{n}\right) \rightarrow 0$. The space $X^{N}$ being linear, we have by 1.4 (b) $N\left(\omega t_{n}\right) \rightarrow 0$. for $|\omega|<1$. In $(-1,1)$ there is a set $E$ of measure greater than $7 / 4$ such that $N\left(\omega t_{n}\right)<\varepsilon$ for almost all $n$ 's for $\omega \in E$. Applying 1.4 (a) and 1.2,A to $\varphi_{n}(\omega)=N\left(\omega t_{n}\right)$ with $n$ sufficiently large, we get for $m$ sufficiently large

$$
N\left(\omega t_{m}\right)<\varepsilon \quad \text { for } \quad|\omega|<1 / 4,
$$

and since $\left|t_{m}\right| \geqslant \varrho$, we infer that $N(t)<\varepsilon$ for $|t|<\varrho / 4$, consequently $N(t)=0$ for $|t|<\varrho / 4$. This implies together with 1.4 (b) that $N(t)=0$ for arbitrary $t$, which is contrary to the hypothesis. Thus $N\left(t_{n}\right) \rightarrow 0$ as $n \rightarrow \infty$ implies $t_{n} \rightarrow 0$. To prove that, conversely, $t_{n} \rightarrow 0$ as $n \rightarrow \infty$ implies $N\left(t_{n}\right) \rightarrow 0$ let us notice that if (b) is not satisfied, then for certain $\bar{t}_{n} \neq 0$ we have $N\left(\bar{t}_{n}\right) \rightarrow 0$ as $n \rightarrow \infty$. By the preceding $\bar{t}_{n} \rightarrow 0$ as $n \rightarrow \infty$. Applying to $N\left(\omega \bar{t}_{n}\right)$ the same argument as formerly to $N\left(\omega t_{n}\right)$ we can prove $N\left(\omega \bar{t}_{m}\right)<\varepsilon$ for $|\omega|<1 / 4$ and $m$ sufficiently large. Let $t_{n} \rightarrow 0$ as $n \rightarrow \infty$. Then since for almost all $n$ we have $t_{n}=\omega_{n} \bar{t}_{m}$ where $\left|\omega_{n}\right|<1 / 4$, we see that $N\left(t_{n}\right)<\varepsilon$.

1.51. The following example shows that without the hypothesis of measurability of $N$ Theorem 1.5 is no longer true. Let $f(z)$ be a complex function of the complex variable discontinuous and satisfying the equations $f\left(z_{1}+z_{2}\right)=f\left(z_{1}\right)+f\left(z_{2}\right), f\left(z_{1} z_{2}\right)=f\left(z_{1}\right) f\left(z_{2}\right)$ for arbitrary $z_{1}, z_{2}$. Let us set $N(t)=|f(t)|$; for real $t$. The space $X^{N}$ is linear, for $N(t+s) \leqslant$ $\leqslant N(t)+N(s), N(s t)=N(s) N(t)$. For the function $N$ none of the condi- 
tions (a), (b), (c) of 1.5 is satisfied, whence we can easily show by aid of 1.3 (a) that there exists no measurable function $M$ such that $X^{N}=X^{M}$ The condition 1.5 (a) does not hold for $N$ for, if it were so, the function $f(z)$ would vanish along the real axis, whence also for every $z$, which is evidently impossible. The condition $1.5(\mathrm{~b})$ is not satisfied for $f(t)=a t+b t i$ for rational $t$ where $a$ and $b$ are real constants. The condition 1.5 (c) is not satisfied. Indeed, if $N$ satisfies the condition $(*)$, the function $f(t)$ is continuous at 0 and since $f\left(t_{1}+t_{2}\right)=f\left(t_{1}\right)+f\left(t_{2}\right)$, we infer that $f(t)=$ $=a t+b t i$ for all real $t$. Hence $f(z)=f(x) \pm f(y) i$ when $z=x+i y$; therefore $f$ would be continuous.

Theorems 1.3, 1.5 explain the importance of the condition (*): Under the hypothesis of measurability of $N$, except the two extreme trivial cases of linear sequence spaces listed in $1.3(\mathrm{a}),(\mathrm{b}), X^{N}$ is a linear space only if the condition (*) is satisfied. If $N$ satisfies the condition (*) (without being measurable), then the cases 1.5 (a), (b) evidently do not hold, whence the condition $(*)$ excludes the above-mentioned extreme cases. The last remarks justify the need of supposing $(*)$ in the sequel.

1.6. Let $N$ satisfy the condition $(*)$; if

(a) there exist constants $O>0, \varepsilon>0$ such that $N(t+s) \leqslant O[N(t)+N(s)]$ for $|t|<\varepsilon,|s|<\varepsilon$

(b) for every $\omega$ there are constants $D_{\omega}>0, \varepsilon_{\omega}>0$ such that $N(\omega t) \leqslant$ $\leqslant D_{\omega} N(t)$ for $|t|<\varepsilon_{\omega}$ then

(c) for every $\varrho>0$ there exists $D>0, \delta>0$ such that $N(\omega t) \leqslant D N(t)$ for $|t|<\delta,|\omega|<\varrho$.

Let us define $M$ as follows:

$$
M(t)=\left\{\begin{array}{ccc}
\varlimsup_{s \rightarrow t} N(s) & \text { as } & |t|<\varepsilon, \\
1 & \text { ass } & |t| \geqslant \varepsilon .
\end{array}\right.
$$

Continuity at 0 of the function $N$ implies, together with (a), $M(t)<\infty$ for $|t|<\varepsilon . M$ is a measurable function equivalent to $N$. To prove that $M \sim N$ let us observe that $M(t) \geqslant N(t)$ in some neighbourhood of 0 The inequality $N(s) \leqslant O[N(t)+N(s-t)]$ satisfied for $|t|<\varepsilon,|s-t|<\varepsilon$ implies $M(t)=\varlimsup_{\lim } N(s) \leqslant C[N(t)+\lim N(s-t)]=C N(t)$ for $|t|<\varepsilon$. It is sufficient to apply 1.12 .

Let us suppose that our theorem does not hold. Then there exist $t_{n} \neq 0, t_{n} \rightarrow 0$ as $n \rightarrow \infty$ and $\omega_{n}$ such that $\left|\omega_{n}\right|<\varrho, N\left(\omega_{n} t_{n}\right) / N\left(t_{n}\right) \rightarrow \infty$ as $n \rightarrow \infty$. Let us observe that the condition (*) implies $N\left(t_{n}\right) \neq 0$, since $t_{n} \neq 0$. Let us define for $n=1,2, \ldots$ and $|\omega|<\varrho$ the functions

$$
\varphi_{n}(\omega)=M\left(\omega t_{n}\right) / M\left(t_{n}\right) \text {. }
$$

We have $M\left(t_{n}\right) \neq 0$, for $M \sim N$. The definition of $M$ implies that the conditions (a), (b) remain valid when $N$ is replaced by $M$. It follows that for $n$ sufficiently large the functions $\varphi_{n}(\omega)$ satisfy the inequality (+) of 1.2 , with some constant $K$ and $\delta=\varrho, E=(-\delta, \delta)$. Applying (b) to $M$ we deduce that the sequence $\varphi_{n}(\omega)$ is bounded for $|\omega|<\varrho$; moreover the functions $\varphi_{n}(\omega)$ are measurable. By $1.2, \mathrm{~B}$ we have $\varphi_{n}(\omega) \leqslant L$ for $|\omega|<\varrho / 4$ and sufficiently large $n$. Hence and from $M \sim N$ we get for almost all $n$

$$
N\left(\omega_{n} t_{n}\right) / N\left(t_{n}\right)<L^{\prime}
$$

with some constant $L^{\prime}>0$, which leads to a contradiction.

1.61. Concerning 1.6 let us notice that there exist non-measurable functions satisfying the conditions (*), 1.6, (a), (c). As an example we may take an arbitrary non-measurable function $N$ satisfying the conditions $a t \leqslant N(t) \leqslant b t$ for $t \geqslant 0$ where $0<a<b, N(t)=N(-t)$ for $t<0$. The non-measurable functions $N$, however, may always be eliminated in the investigation of linear spaces $X^{N}$ provided that the condition (*) be satisfied. Indeed,

Let the function $N$ satisfy the condition $(*)$ and $1.6(\mathrm{a}),(\mathrm{b})$; then there exists a function $M$ equivalent to $N$ continuous and increasing for $t \geqslant 0$ and such that $M(t)=M(-t)$.

To prove this let us choose a positive integer $m$ so that $N(t) \leqslant 1$ for $0 \leqslant t \leqslant 1 / m$ and let $\varepsilon_{n}$ be a decreasing sequence such that $0<\varepsilon_{n} \leqslant$ $\leqslant \sup _{0 \leqslant s \leqslant 1 / n} N(s)$ for $n>m$. This is possible, for $N(t)$ is continuous at 0 and

$N(t) \neq 0$ for $t \neq 0$. Let us define first $M(t)$ for $t \geqslant 0$ as follows:

$$
\begin{aligned}
& M(0)=0, \\
& M(1 / n)=\sup _{0 \leqslant s \leqslant 1 / n} N(s)+\varepsilon_{n},
\end{aligned}
$$

$M(t)$ is equal to the linear function for $1 /(n+1) \leqslant t \leqslant 1 / n$, if $n \geqslant m$,

$M(t)$ is equal to an arbitrary continuous function increasing for $t>1 / m$ provided that it is chosen so that the continuity of $M$ is preserved at the point $t=1 / \mathrm{m}$.

For $t<0$ we set $M(t)=M(-t)$. The equivalence of $M$ and $N$ results from the following inequalities, which result from 1.6 (c):

$$
\begin{aligned}
\sup _{0 \leqslant s \leqslant 1 / n} N(s) & \leqslant D N(t), \quad N\left(\frac{1}{n+1}\right) \geqslant \frac{N(t)}{D} \quad \text { for } \quad \frac{1}{n+1}<t<\frac{1}{n}, \\
M(t) & \leqslant M\left(\frac{1}{n}\right) \leqslant 2 \sup _{0 \leqslant s \leqslant 1 / n} N(s) \leqslant 2 D N(t), \\
M(t) & \geqslant M\left(\frac{1}{n+1}\right) \geqslant \sup _{0 \leqslant s \leqslant 1 /(n+1)} N(s) \geqslant N\left(\frac{1}{n+1}\right) \geqslant \frac{N(t)}{D}
\end{aligned}
$$


for $1 /(n+1) \leqslant t<1 / n$. In all these inequalities $n \geqslant m, n>1 / \delta$ and $\delta, D$ are constants involved in $\mathbf{1 . 6}$ (c) when $\varrho=2$. We can suppose beforehand about $N$ that $N(-t)=N(t)$ for $t>0$, for every function $N$ satisfying the condition $1.6(\mathrm{~b})$ is equivalent to $\bar{N}(t)=N(|t|)$; consequently $M \sim N$.

1.62. Let $N$ be non-decreasing for $t \geqslant 0$ and let $N(t)=N(-t)$ for all $t$; then the conditions 1.6 (a), (b) are equivalent to

(+) $\quad N(2 t) \leqslant K N(t)$ for some $K$ in a neighbourhood of 0 .

Setting $t=s$ in 1.6 (a) we get $\left({ }^{+}\right)$. Conversely let the condition $(+)$ be satisfied. The function $N$ being monotone and even, we have for sufficiently small $t, s$

$$
\begin{aligned}
N(|t+s|) & \leqslant N(|t|+|s|) \leqslant N(2 \max (|t|,|s|)) \\
& \leqslant K N(\max (|t|,|s|)) \leqslant K[N(|t|)+N(|s|)] .
\end{aligned}
$$

If $|\omega| \leqslant 1$, then $1.6(\mathrm{~b})$ is satisfied with $D_{\omega}=1$ and arbitrary $\varepsilon_{\omega}$. Let $|\omega|>1$; choose a positive integer $n$ so that $2^{n-1} \leqslant|\omega|<2^{n}$. It follows from $(+)$ that in some neighbourhood of 0

$$
N(|\omega||t|) \leqslant N\left(2^{n} \frac{|\omega|}{2^{n}}|t|\right) \leqslant K^{n} N\left(\frac{|\omega|}{2^{n}}|t|\right) \leqslant K^{n} N(|t|) .
$$

1.7. THEORTM 1 . Let $N$ satisfy the condition (*). The necessary and sufficient condition that $X^{N}$ be a linear space is that the conditions 1.6 (a), (c) be satisfied. The function $N$ may always be replaced by a continuous even function $M$ increasing for $t \geqslant 0$ and equivalent to $N$, i. e. such that $X^{M}=X^{N}$.

1.8. Now we shall discuss the possibility of introducing the norm in the linear spaces $X^{N}$. We remember that, in a linear space, the norm of type $F$ is a functional \|\| satisfying the conditions 1 ) $\|x\| \geqslant 0$, 2) $\|x\|=0$ if and only if $x=0,3)\|x+y\| \leqslant\|x\|+\|y\|, 4)\|x\|=\|-x\|, 5)$. the product $\omega x$ where $\omega$ is real is continuous with respect to the norm in both variables jointly. A linear space provided with an $F$-norm will be called the $F^{*}$-space ; if, moreover, the axiom of completeness is satisfied it will be called the $F$-space.

Let the space $X^{N}$ be linear; we shall say that the sequence $x_{n}$ of elements of $X^{N}$ is $\varrho$-convergent to $x_{0}$ (in symbols $x_{n} \stackrel{\varrho}{\rightarrow} x_{0}$ as $n \rightarrow \infty$ ) if $\varrho\left(x_{n}-x_{0}\right) \rightarrow 0$ for $n \rightarrow \infty$. Suppose that an $F$-norm \|\| is defined in $X^{N}$; the convergence with respect to the norm \|\| will be called equivalent to the convergence $\varrho$ if the relation $\left\|x_{n}-x_{0}\right\| \rightarrow 0$ as $n \rightarrow \infty$ implies $\varrho\left(x_{n}-x_{0}\right) \rightarrow 0$ for $n \rightarrow \infty$, and conversely. Let us observe that if the convergence with respect to the norm is equivalent to the convergence $\varrho$, then $\|x\|=0$ if and only if $\varrho(x)=0$.
1.81. Let $N$ satisfy the condition (*) and let the space $X^{N}$ be linear. If:

(a) $x \in X^{N}, \omega_{n} \rightarrow 0$, then $\omega_{n} x \stackrel{\varrho}{\rightarrow} 0$ as $n \rightarrow \infty$,

(b) $x_{n} \in X^{N}$, the sequence $\omega_{n}$ is bounded and $x_{n} \stackrel{\circ}{\rightarrow} 0$ as $n \rightarrow \infty$, then $\omega_{n} x_{n} \stackrel{\stackrel{o}{\rightarrow}}{\rightarrow} 0$.

For the proof let us notice that if $x=\left\{t_{n}\right\} \in X^{N}$, then, by 1.6 (c),

$$
\begin{gathered}
\sum_{n=p}^{\infty} N\left(\omega t_{n}\right) \leqslant D \sum_{n=p}^{\infty} N\left(t_{n}\right) \quad \text { when } \sum_{n=p}^{\infty} N\left(t_{n}\right) \leqslant \delta,|\omega|<r, \\
\varrho(\omega x) \leqslant D \varrho(x) \text {. when } \varrho(x)<\delta,|\omega|<r,
\end{gathered}
$$

where $D, \delta$ are constants of 1.6 (c) and the constant $\varrho$ appearing in 1.6 (c) is denoted by $r$. The first of these inequalities and the continuity of $N$ at 0 imply (a), the second inequality implies (b).

1.811. Let $M \sim N$; then $\varrho_{M}\left(x_{n}\right) \rightarrow 0$ as $n \rightarrow \infty$ implies $\varrho_{N}\left(x_{n}\right) \rightarrow 0$ as $n \rightarrow \infty$, and conversely.

This follows immediately from the definition of equivalent functions.

1.82. Let the function $N$ be non-decreasing for $t \geqslant 0, N(t)=N(-t)$ for all $t$ and let it satisfy the condition $(*)$; let the space $X^{N}$ be linear. One can define in $X^{N}$ an $F$-norm so that convergence with respect to the norm is equivalent to the convergence $\varrho$. With this definition of norm $X^{N}$ is an $\mathrm{F}^{\mathrm{H}}$-space.

By 1.6 (c) and 1.81 (a) we have $\varrho(x / \varepsilon) \rightarrow 0$ as $0<\varepsilon \rightarrow \infty$, whence there exist $\varepsilon>0$ satisfying the inequality

$$
\varrho(x / \varepsilon)<\varepsilon .
$$

Let us define the norm $\|x\|=\inf \varepsilon$, the infimum being extended over the set of the $\varepsilon>0$ satisfying $(+)$. We shall verify that\| $\|$ satisfies all the axioms of $F$-norms. Let us observe first that $\varrho(x) \leqslant \varrho(x / \varepsilon) \leqslant \varepsilon$ when $\varrho(x / \varepsilon) \leqslant \varepsilon, 0<\varepsilon \leqslant 1$. We shall prove that

$(++) \quad\left\|x_{n}\right\| \rightarrow 0$ as $n \rightarrow \infty$ implies $\varrho\left(x_{n}\right) \rightarrow 0$ as $n \rightarrow \infty$, and conversely.

Indeed, if $\left\|x_{n}\right\| \rightarrow 0$ as $n \rightarrow \infty$, then $\varrho\left(x_{n} / \varepsilon\right)<\varepsilon$ for $0<\varepsilon<1$ and large $n$, whence $\varrho\left(x_{n}\right)<\varepsilon$. Conversely let $\varrho\left(x_{n}\right) \rightarrow 0$ as $n \rightarrow \infty$ : then in virtue of 1.81 (b) we have $\rho\left(x_{n} / \varepsilon\right)<\varepsilon$ for large $n$, whence $\left\|x_{n}\right\|<\varepsilon$ for large $n$. The symmetry $\|x\|=\|-x\|$ follows directly from the fact that $N$ is even; $\|0\|=0$ is obvious. If $\|x\|=0$, then applying $(++)$ to the sequence $x, x, \ldots$ we get $\varrho(x)=0, i$. e. $x=0$. To prove the triangle inequality we may suppose that $\|x\|>0\|y\|>0$. Given $\delta>0$, there exist $\varepsilon>0, \eta>0$, satisfying $(+)$ and $\varrho(y / \eta)<\eta$ respectively, such that $\varepsilon<\|x\|+\delta, \eta<\|y\|+\delta$. Then 


$$
\begin{aligned}
N\left(\frac{t_{n}+s_{n}}{\varepsilon+\eta}\right) & =N\left(\frac{\left|t_{n}+s_{n}\right|}{\varepsilon+\eta}\right) \leqslant N\left(\frac{\left|t_{n}\right|+\left|s_{n}\right|}{\varepsilon+\eta}\right) \leqslant N\left(\frac{\left|t_{n}\right|}{\varepsilon} \frac{\varepsilon}{\varepsilon+\eta}+\frac{\left|s_{n}\right|}{\eta} \frac{\eta}{\varepsilon+\eta}\right) \\
& \leqslant \sup \left(N\left(\frac{\left|t_{n}\right|}{\varepsilon}\right), N\left(\frac{\left|s_{n}\right|}{\eta}\right)\right) \leqslant N\left(\frac{\left|t_{n}\right|}{\varepsilon}\right)+N\left(\frac{\left|s_{n}\right|}{\eta}\right),
\end{aligned}
$$

whence

$$
\varrho\left(\frac{x+y}{\varepsilon+\eta}\right) \leqslant \varrho\left(\frac{x}{\varepsilon}\right)+\varrho\left(\frac{y}{\eta}\right)<\varepsilon+\eta<\|x\|+\|y\|+2 \delta
$$

which implies $\|x+y\| \leqslant\|x\|+\|y\|+2 \delta$.

The continuity of $\omega x$ with respect to the variables $(\omega), x$ follows by 1.81 and $(++)$. Finally, $(++)$ implies the equivalence of the $\varrho$-convergence to the convergence with respect to the norm. To prove the axiom of completeness let us choose a continuous function $M$ satisfying (*) equivalent to $N$ (this is possible in virtue of 1.61). Let $\left\|x_{p},-x_{q}\right\| \rightarrow 0$ as $p, q \rightarrow \infty$, whence because of $1.811 \varrho_{M}\left(x_{p}-x_{q}\right) \rightarrow 0$ as $p, q \rightarrow \infty$. It follows $t_{n}^{(r)} \rightarrow t_{n}^{(0)}$ as $r \rightarrow \infty$ for $n=1,2, \ldots$ (we have set here $x_{r}=\left\{t_{n}^{(r)}\right\}$ ). For sufficiently large $p, q$ we have $\varrho_{M}\left(x_{p}-x_{q}\right)<\varepsilon$, whence by the continuity of $M$

$$
\varrho_{M}\left(x_{p}-x_{0}\right) \leqslant \varepsilon,
$$

where $x_{0}=\left\{t_{n}^{(0)}\right\}$, i. e. $\varrho_{M}\left(x_{p}-x_{0}\right) \rightarrow 0$ as $p \rightarrow \infty$ and consequently $\left\|x_{p}-x_{0}\right\| \rightarrow 0$ as $p \rightarrow \infty$. Since $x_{p}-x_{0} \in X^{N}$ for large $p, x_{p} \in X^{N}$, whence $x_{0} \in X^{N}$. Putting together 1.82 and 1.61 and taking into account 1.811 we get

THEOREM 2. Each $X^{N}$ linear sequence space forms an $T^{H}$-space if $N$ satisfies the condition $(*)$; the $F$-norm may be defined so that the $\varrho^{-c o n v e r-}$ gence is equivalent to the convergence with respect to the norm.

1.9. If $X^{N}$ is a locally convex $F$-space, $N$ satisfies the condition (*) and norm-convergence implies $\varrho$-convergence, then $N$ is equivalent. to a continuous even convex function satisfying the condition (*).

By a known theorem of Banach the norm in $X^{N}$ is equivalent to the norm of theorem 2, whence the convergence with respect to the norm is equivalent to the convergence $\varrho$. We may suppose by 1.61 that $N$ is continuous, even and increasing for $t \geqslant 0$. The local convexity of $X^{N}$ implies the existence of $\varepsilon>0$ such that $\varrho\left(x_{k}\right)<\varepsilon$ for $k=1,2, \ldots, n$ implies $\left.\varrho\left(x_{1}+x_{2}+\ldots+x_{n}\right) / n\right)<1$. Let us denote by $e_{m}$ the sequence $\left\{t_{7_{i}}^{m}\right\}, t_{7_{0}}^{m}=1$ for $k=m,=0$ for $k \neq m$. Writing for any positive integer $p, x_{k}=$ $t\left(e_{k}+e_{k+n}+\ldots+e_{k+(p-1) n}\right)$, we get $\varrho\left(x_{k}\right)=p N(t)$,

$$
\varrho\left(\frac{x_{1}+x_{2}+\ldots+x_{n}}{n}\right)=p n N\left(\frac{t}{n}\right)
$$

whence for $N(t) \leqslant \varepsilon / p$ we have $N(t / n) \leqslant 1 / p n$. If $0<N(t) \leqslant \varepsilon$ and $p$ is chosen so that $\varepsilon /(p+1)<N(t) \leqslant \varepsilon / p$, then $N(t / n) \leqslant 1 / p n \leqslant(2 / \varepsilon n) N(t)$. Given $\varrho>0$ let us choose $q \geqslant 1$ so that $N(t / q)<\varepsilon$ for $|t| \leqslant \varrho$. We have

$$
N(q t) \leqslant D_{q} N(t) \text { for } \quad|t| \leqslant \varrho .
$$

Indeed, this inequality is true with some constant for $|t|<\delta_{q}$ in virtue of $1.6(\mathrm{c})$, and since $\inf N(t)>0, \sup N(q t)<\infty$ in $\left\langle\delta_{q}, \varrho\right\rangle$, we may choose $D_{q}>0$ so that $(+)$ is satisfied in the whole of the interval $|t| \leqslant \varrho$. By the inequality proved previously

$$
N\left(\frac{t}{n}\right)=N\left(q \frac{|t|}{q n}\right) \leqslant D_{q} N\left(\frac{|t|}{q n}\right) \leqslant \frac{1}{n} \frac{2}{\varepsilon} D_{q} N(t) \quad \text { as } \quad|t| \leqslant \varrho .
$$

If $0<\omega \leqslant 1$ let us choose $n$ so that $1 /(n+1)<\omega \leqslant 1 / n$. As $1 / n \leqslant 2 \omega$, setting $K_{\rho}=4 D_{q} / \varepsilon$ we get

$$
(++) \quad . \quad N(\omega t) \leqslant K_{e} \omega N(t) \quad \text { for } \quad 0 \leqslant \omega \leqslant 1,|t| \leqslant \varrho \text {. }
$$

Let us write

$$
P(t)=\sup _{0<\omega \leqslant 1} \frac{N(\omega t)}{\omega}
$$

By $(++)$ it follows that $0 \leqslant P(t)<\infty$ for each $t$; moreover, $P(\alpha t) \leqslant$ $\leqslant \alpha P(t)$ for $0 \leqslant \alpha \leqslant 1$. By $(++) P(t) \leqslant K_{1} N(t)$ for $|t| \leqslant 1$; moreover $P(t) \geqslant N(t)$, whence $P \sim N$. Let us set $Q(t)=P(t) / t$ for $t>0$; this function is non-decreasing on the half axis $t>0$, for we have for $0<t^{\prime}<t^{\prime \prime}$

$$
Q\left(t^{\prime}\right)=\frac{P\left(t^{\prime}\right)}{t^{\prime}}=\frac{P\left(\frac{t^{\prime}}{t^{\prime \prime}} t^{\prime \prime}\right)}{t^{\prime}} \leqslant \frac{\frac{t^{\prime}}{t^{\prime \prime}} P\left(t^{\prime \prime}\right)}{t^{\prime}}=\frac{P\left(t^{\prime \prime}\right)}{t^{\prime \prime}}=Q\left(t^{\prime \prime}\right) .
$$

Let us now define

$$
M(t)=\int_{0}^{t} Q(s) d s \quad \text { for } \quad t \geqslant 0, \quad M(t)=M(-t) \quad \text { for } \quad t<0 .
$$

The function $M$ is evidently convex, moreover $N \sim M$. The equivalence follows by the inequalities

$$
M(t) \leqslant t Q(t)=P(t), \quad M(t) \geqslant \int_{t / 2}^{t} Q(s) d s=\frac{t}{2} Q\left(\frac{t}{2}\right)=P\left(\frac{t}{2}\right) \text { for } t \geqslant 0,
$$

$P\left(\frac{t}{2}\right) \geqslant N\left(\frac{t}{2}\right) \geqslant \frac{1}{D} N(t) \quad$ in a neighbourhood of 0 . 
1.91. Let the space $X^{N}$ be linear. If the function $N$ satisfies the condition (*) and $N$ is equivalent to a convex function, then $X^{N}$ is a B-space with norm-convergence equivalent to $\varrho$-convergence.

Let the function $M$ be convex and equivalent to $N$. The continuity of $N$ at 0 implies the same for the function $M$, whence $M$ is continuous everywhere. We may suppose that $M(t)=M(-t)$ for $t \geqslant 0$; moreover $M(0)=0$ for $N(0)=0$ and $M \sim N$. The function $M$ satisfies the condition (*). Indeed, if $t_{n} \rightarrow 0$ as $n \rightarrow \infty$, then $M\left(t_{n}\right) \rightarrow 0$; if $M\left(t_{n}\right) \rightarrow 0$, then $t_{n \rightarrow 0}$ as $n \rightarrow \infty$. In the contrary case we would infer from the continuity and convexity of $M$ that $M(t)=0$ in some neighbourhood of 0 , whence also $N(t)$ in a neighbourhood of 0 , which is impossible, for $N$ satisfies the condition $(*)$. Let $W$ denote the set of the elements $x$ satisfying the condition $\varrho_{M}(x) \leqslant 1$. From the properties of $M$ it follows that the set $W$ is convex and symmetric with respect to 0 . By 1.82 there exists in $X^{N}=X^{M}$ an $F$-norm, say \|\|$^{*}$, such that $\varrho_{M}$-convergence is equivalent to the convergence with respect to \|\|$^{*}$. Thus choosing a sufficiently small $r>0$ we deduce from $\|x\|^{*} \leqslant r$ that $\varrho_{M}(x) \leqslant 1, i$.e 0 is an inner point of $W$ in the \|\|$^{*}$-normed space $X^{N}$. The set $W$ is also bounded; in fact $M(\omega t)=$ $=M(|\omega||t|) \leqslant|\omega| M(|t|)$ as $|\omega| \leqslant 1$, whence $\varrho_{M}(\omega x) \leqslant|\omega| \varrho_{M}(x)$ for $|\omega| \leqslant 1$. From the above properties of $W$ it follows by a known theorem that the norm \|\|$^{*}$ is equivalent to a $B$-norm. Let us observe that the corresponding $B$-norm may be obtained as follows. Let us set $\|x\|=\inf k$, the infimum being taken over the set of the $k>0$ satisfying the inequality $\left.o_{M}(x / k) \leqslant 1^{1}\right)$. The axioms of the norm are easily verified. One can also prove directly the equivalence of the convergence $\varrho_{M}$ and the convergence with respect to \|\| , which implies in turn the axiom of completeness.

1.92. As a corollary to 1.9 and 1.91 and taking into account that for even functions $M$ non-decreasing for $M \geqslant 0$ the condition 1.6 (a) is equivalent to $1.62(+)$, we deduce

THEOREM 3. Let the function $N$ satisfy the condition (*), then

(a) $X^{N}$ is a Banach space if and only if the function $N$ is equivalent to a continuous convex even function $M$ vanishing only at 0 and satisfying the inequality $1.62(+)$,

(b) if $X^{N}$ is a $B_{0}$-space, $X^{N}$ is a B-space.

Here norm-convergence is always to understand as equivalent to o-convergence.

1) This method of the introduction of the norm in $X^{N}$ is known (see [2])
2.1. Throughout this section we suppose that $N$ and $M$ are Baire functions; this hypothesis will not be mentioned in the subsequent considerations. We shall use the notation

$$
\varrho_{N}(x)=\int_{0}^{1} N(x(t)) d t
$$

moreover, the subscript $N$ will be omitted when there is no doubt about the considered function $N$. Since $N$ is a Baire function, the function $N(x(t))$ is measurable when $x(t)$ is measurable. The set of those $x$ for which $\rho_{N}(x)<\infty$ will be denoted by $X^{N}$. Saying " $N$ satisfies the condition (*)" we shall always mean the condition 1.1. $(*)$. We shall also often need the following condition:

(o) $\quad N\left(t_{n}\right) \rightarrow \infty$ as $n \rightarrow \infty$ if and only if $\left|t_{n}\right| \rightarrow \infty$ as $n \rightarrow \infty$.

The condition (o) obviously implies that $N$ is bounded in every finite interval.

The functions $N$ and $M$ will be said to be equivalent if they have the following properties:

There exist constants $A>0, B>0, K>0, r>0$ such that the following inequalities are satisfied:

$(+) \quad N(t) \leqslant B M(t)$ for $M(t) \geqslant r, \quad M(t) \leqslant A N(t)$ for $N(t) \geqslant r$, $(++) \quad N(t) \leqslant K \quad$ for $\quad M(t)<r, \quad M(t) \leqslant K \quad$ for $\quad N(t)<r$.

For this definition of equivalence we shall use the same notation as in section 1 to denote the equivalent functions: $N \sim M$.

2.12. Let $N$ and $M$ satisfy the condition (o); then $N \sim M$ if and only if simultaneously $N(t) \leqslant B M(t), M(t) \leqslant A N(t)$ for sufficiently large $|t|$.

2.13. Let $M(t, s)$ and $N(t, s)$ be two non-negative Baire functions of the variables $t, s$, defined for all real $t, s . A$ necessary and sufficient condition that for arbitrary functions $x, y$ the inequality

$$
\int_{0}^{1} M(x(t), y(t)) d t<\infty
$$

imply

$$
\int_{0}^{1} N(x(t), y(t)) d t<\infty
$$

is that for certain constants $O>0, K>0, r>0$ the following inequalities be satisfied:

$\begin{array}{llll}(+) & N(t, s) \leqslant C M(t, s) & \text { as } & M(t, s) \geqslant r, \\ (++) & N(t, s) \leqslant K & \text { as } & M(t, s)<r .\end{array}$

Sufficiency is evident. 
Necessity. Let us supppose that $(+)$ is not satisfied. Then there exist $t_{n}, s_{n}$ such that

$$
\sum_{n=1}^{\infty} \frac{1}{M\left(t_{n}, s_{n}\right)} \leqslant 1, \quad N\left(t_{n}, s_{n}\right) \geqslant n M\left(t_{n}, s_{n}\right) \quad \text { for } \quad n=1,2, \ldots
$$

Let $\delta_{1}, \delta_{2}, \ldots$ denote disjoint intervals in $(0,1)$ with lengths

$$
\frac{1}{1^{2} M\left(t_{1}, s_{1}\right)}, \frac{1}{2^{2} M\left(t_{2}, s_{2}\right)}, \cdots
$$

Let

$$
\begin{aligned}
& x(s)=\left\{\begin{array}{lll}
t_{n} & \text { for } & s \in \delta_{n}, n=1,2, \ldots, \\
0 & \text { for } & s \in(0,1)-\bigcup_{1}^{\infty} \delta_{n},
\end{array}\right. \\
& y(s)=\left\{\begin{array}{lll}
s_{n} & \text { for } & s \in \delta_{n}, n=1,2, \ldots, \\
0 & \text { for } & s \in(0,1)-\bigcup_{1}^{\infty} \delta_{n} .
\end{array}\right.
\end{aligned}
$$

Then

$$
\int_{0}^{1} M(x(s), y(s)) d s=\sum_{n=1}^{\infty} \frac{1}{n^{2}}, \quad \int_{0}^{1} N(x(s), y(s)) d s \geqslant \sum_{n=1}^{\infty} \frac{1}{n}=\infty,
$$

contrary to hypothesis. Let us now suppose that $(+)$ is not satisfied. There exist $t_{n}, s_{n}$ such that $N\left(t_{n}, s_{n}\right) \rightarrow \infty$ for $n \rightarrow \infty, M\left(t_{n}, s_{n}\right) \leqslant r$. Let us choose positive $a_{n}$ so that

$$
\sum_{n=1}^{\infty} a_{n} \leqslant 1, \quad \sum_{n=1}^{\infty} a_{n} N\left(t_{n}, s_{n}\right)=\infty,
$$

and then disjoint intervals $\delta_{n}$ in $(0,1)$ with lengths $a_{n}$; next we define the functions $x, y$ by aid of $\left(\begin{array}{c}++ \\ +\end{array}\right)$. We obtain

$$
\int_{0}^{1} M(x(s), y(s)) d s<\infty, \quad \int_{0}^{1} N(x(s), y(s)) d s=\infty
$$

which leads to a contradiction.

Setting $M(t, s)=M(t), N(t, s)=N(t)$ for arbitrary $t, s$ we deduce from 2.13 that

2.14. A necessary and sufficient condition that the inequality $\varrho_{M}(x)<\infty$ imply $\varrho_{N}(x)<\infty$ is the existence of constants $B>0, K>0, r>0$ such that

$$
\begin{array}{lll}
N(t) \leqslant B M(t) & \text { for } & M(t) \geqslant r, \\
N(t) \leqslant K & \text { for } & M(t)<r .
\end{array}
$$

2.2. (a) $X^{N}=X^{M}$ if and only if $N \sim M$;

(b) $X^{N}$ is identical with the space of all measurable functions if and only if $M$ is bounded in $(-\infty, \infty)$.

$A d$ (a). It immediately follows from 2.14 and the definition of equivalence.

$A d$ (b). Sufficiency is trivial.

Necessity. If $N$ is unbounded, then there exist $t_{n}$ such that

$$
N\left(t_{n}\right) \rightarrow \infty \quad \text { as } \quad n \rightarrow \infty, \quad \sum_{n=1}^{\infty} \frac{1}{N\left(t_{n}\right)} \leqslant 1 .
$$

Let us choose disjoint intervals $\delta_{n}$ in $(0,1)$ with lengths $1 / N\left(t_{n}\right)$ and let us set

$$
x(s)=\left\{\begin{array}{lll}
t_{n} & \text { for } & s \in \delta_{n}, n=1,2, \ldots, \\
0 & \text { for } & s \in(0,1)-\bigcup_{n=1}^{\infty} \delta_{n} .
\end{array}\right.
$$

Obviously $\varrho_{N}(x)=\infty$ and $X^{N}$ does not contain all measurable functions.

2.3. The following conditions are necessary and sufficient that $X^{N}$ be a linear space:

(a) There exist constants $O>0, K>0, r>0$ such that

$$
N(t+s) \leqslant C(N(t)+N(s)) \text { for } N(t)+N(s) \geqslant r ;
$$

(a') $N(t+s) \leqslant K \quad$ for $\quad N(t)+N(s)<r$

(b) for each w there exist constants $D_{\omega}>0, K_{\omega}>0, r_{\omega}>0$ such that

$$
N(\omega t) \leqslant D_{\omega} N(t) \quad \text { for } \quad N(t) \geqslant r_{\omega} ;
$$

$\left(\mathrm{b}^{\prime}\right) N(\omega t) \leqslant K_{\omega}$ for $N(t)<r_{\omega}$.

To prove (a), (a') let us write $M(t, s)=N(t)+N(s), N(t, s)=N(t+s)$. As $\varrho_{N}(x)<\infty, \varrho_{N}(y)<\infty$ implies $\varrho_{N}(x+y)<\infty$, it is sufficient to apply 2.13 to $M(t, s), N(t, s)$. To prove (b), ( $\left.\mathrm{b}^{\prime}\right)$ let us make use of 2.14 replacing $N(t)$ by $N(\omega t)$ and $M(t)$ by $N(t)$. Then we take into account that $\varrho_{N}(x)<\infty$ implies $\varrho_{N}(\omega x)<\infty$ for each $\omega$.

2.31. Let $N$ satisfy the conditions $2.3(\mathrm{~b})$ and $\left(\mathrm{b}^{\prime}\right)$ and let the sequence $N\left(t_{n}\right)$ be bounded; then for every $\omega$ the sequence $N\left(\omega t_{n}\right)$ is also bounded. Suppose that $N\left(t_{n}\right) \leqslant L$ for $n=1,2, \ldots$ If $N\left(t_{n}\right) \geqslant r_{\omega}$ then $N\left(\omega t_{n}\right) \leqslant D_{\omega} L$; if $N\left(t_{n}\right)<r_{\omega}$, then $N\left(\omega t_{n}\right) \leqslant K_{\omega}$.

2.4. Let $N$ satisfy the conditions $(\mathrm{a}),\left(\mathrm{a}^{\prime}\right),(\mathrm{b}),\left(\mathrm{b}^{\prime}\right)$ of 2.3 ; then for every $\varrho>0$ there exist positive constants $D, F$ such that

$$
\begin{array}{lll}
N(\omega t) \leqslant D N(t) & \text { for } & N(t) \geqslant r_{\varrho},|\omega|<\varrho, \\
N(\omega t) \leqslant F & \text { for } & N(t)<r_{\varrho},|\omega|<\varrho .
\end{array}
$$


Let us suppose that $\left|\omega_{n}\right|<\varrho, N\left(t_{n}\right) \rightarrow \infty$ as $n \rightarrow \infty$ where $N\left(t_{n}\right)>0$, and that $N\left(\omega_{n} t_{n}\right) / N\left(t_{n}\right) \rightarrow \infty$ as $n \rightarrow \infty$. As in 1.6 we define functions for $n=1,2, \ldots$ and $|\omega|<\varrho$

$$
p_{n}(\omega)=\frac{N\left(\omega 4 t_{n}\right)}{N\left(t_{n}\right)} .
$$

It follows from 2.3 (b) that for every $(\omega)$ in $(-\varrho, \varrho)$ the sequence $\varphi_{n}(\omega)$ is bounded. Since $N\left(t_{n}\right) \rightarrow \infty$, it follows from 2.31. for $\omega \neq 0$ that $N\left(\omega t_{n}\right) \rightarrow \infty$ and, the functions $N\left(\omega t_{n}\right)$ being measurable, there exists in $(-\varrho, \varrho)$ a measurable set $E$ of measure $>\frac{7}{4} \varrho$ such that $N\left(\omega 4 t_{n}\right) \geqslant r$ for $\omega \in E$ and sufficiently large $n$. Thus by 2.3 (a) it follows that for $\left(\omega_{1},()_{2} \in E\right.$ and sufficiently large $n$

$$
\varphi_{n}\left(\omega_{1}+\omega_{2}\right) \leqslant C\left[\varphi_{n}\left(\omega_{1}\right)+\varphi_{n}\left(\omega_{2}\right)\right],
$$

whence by $1.2 \mathrm{~B}$ there is a constant $L$ such that

$$
\varphi_{n}\left(\omega t_{n}\right) \leqslant L \quad \text { for } \quad|\omega|<\varrho / 4 \text { and large } n ;
$$

therefore

$$
\frac{N\left(\omega t_{n}\right)}{N\left(t_{n}\right)} \leqslant L \text { for } \quad|\omega|<\varrho \text { and large } n,
$$

in contradiction to the relation $N\left(\omega_{n} t_{n}\right) / N\left(t_{n}\right) \rightarrow \infty$ as $n \rightarrow \infty$.

To prove the second inequality of our proposition let us suppose that for certain $t_{n}, \omega_{n}$ such that $\left|\omega_{n}\right|<\varrho, N\left(t_{n}\right)<r_{\varrho}$ we have $N\left(\omega_{n} t_{n}\right) \rightarrow \infty$. Let us define measurable functions for $n=1,2, \ldots$.

$$
\varphi_{n}(\omega)=N\left(\omega 4 t_{n}\right)
$$

By 2.31 the sequence $p_{n}(\omega)$ is bounded as $\omega \epsilon(-\varrho, \varrho)$. The same argument as in the proof of $1.2, \mathrm{~B}$, with the same notation except that $\delta$ is replaced by $\varrho$, leads to the representation $1.2(++)$, whence by 2.3 (a) there follows $1.2\left({ }_{++}^{+}\right)$(where $K$ is to be replaced by $\left.C^{\prime}\right)$ if $N\left(\omega_{1} 4 t_{n}\right)+$ $+N\left(-\omega_{2} 4 t_{n}\right) \geqslant r$. On the other hand, if $N\left(\omega_{1} 4 t_{n}\right)+N\left(-\omega_{2} 4 t_{n}\right)<r$, then from $2.3\left(a^{\prime}\right)$ we deduce

$$
\varphi_{n}(\omega)=N\left(\omega 4 t_{n}\right) \leqslant K \quad \text { for } \quad|\omega|<\varrho / 4 ;
$$

consequently the sequence $N\left(\omega 4 t_{n}\right)$ is uniformly bounded in $(-\varrho / 4, \varrho / 4)$, whence also the sequence $N\left(\omega t_{n}\right)$ in $(-\varrho, \varrho)$, which is contrary to the fact that $N\left(\omega_{n} t_{n}\right) \rightarrow \infty$ as $n \rightarrow \infty$ where $\left|\omega_{n}\right|<\varrho$.

2.5. Let $X^{N}$ be a linear space different from the space of all measurable functions; then $N$ satisfies the condition (0).
By 2.3 and 2.4 $N(\omega)$ is bounded in $(-\varrho, \varrho)$, whence in every finite interval. Therefore if $N\left(t_{n}\right) \rightarrow \infty$ as $n \rightarrow \infty$, then $\left|t_{n}\right| \rightarrow \infty$ as $n \rightarrow \infty$. Conversely, suppose that $\left|t_{n}\right| \rightarrow \infty$ as $n \rightarrow \infty$, and let $r>0$ be given. If we had $N\left(t_{n}\right)<r$ for $n=1,2, \ldots$, then, by 2.4, $N\left(\omega t_{n}\right) \leq=\max \left(D_{r_{1}} r, F\right)$ for $|\omega|<1, n=1,2, \ldots$, whence $N(t)$ would be bounded in $(-\infty, \infty)$, which is contrary to 2.2 (b).

2.6. Let $N$ satisfy the condition (o) and let $X^{N}$ be a linear space. Then there exists a function $M$ equivalent to $N$, continuous increasing for $t \geqslant 0$ such that $M(t)=M(-t)$ and satisfying the conditions (o), (*).

Let us observe first that we may suppose that $N(t)=N(-t)$ for arbitrary $t$. Indeed, the condition (o) being satisfied by $N$, it follows by 2.3 (b), (b') that $N \sim \bar{N}$ where $\bar{N}(t)=N(|t|)$.

Let $m$ be the smallest positive integer such that $\sup N(s)>0$. Let us set $M(n)=\varepsilon_{n} \sup N(s)$ for $n \geqslant m$ where $0<\varepsilon_{n}<1$ are chosen to form us set $M(n)=\varepsilon_{n} \cos _{0 \leqslant n}$. an increasing sequence. We define the value 0 at $t=0$, in the interval $0 \leqslant t \leqslant m$; for $n \leqslant t \leqslant n+1$ with $n \geqslant m$, the function $M(t)$ is equal to the linear function, $M(t)=M(-t)$ for $t<0$. $M$ obviously satisfies the condition (*); the condition (0) being satisfied by $N$, it follows that $\sup _{0 \leq s \leq n} N(s) \rightarrow \infty$ as $n \rightarrow \infty$, which implies that $M$ satisfies the condition $(0)$ too. For $n \leqslant t \leqslant n+1$ where $n \geqslant m$ we have

$$
\begin{gathered}
M(t) \leqslant M(n+1)=\varepsilon_{n+1} \sup _{0 \leqslant s \leqslant n+1} N(s)=\varepsilon_{n+1} \sup _{0 \leqslant \omega \leqslant(n+1) / t} N(\omega t) \leqslant \\
\leqslant \varepsilon_{n+1} \sup _{0 \leqslant \omega \leqslant 2} N(\omega t), \\
M(t) \geqslant M(n) \geqslant \varepsilon_{n} N(n) \geqslant \varepsilon_{m} N(n) .
\end{gathered}
$$

And since by 2.4 and the condition (0) for $N$ the inequalities

$$
\begin{gathered}
\sup _{0 \leqslant \omega \leqslant 2} N(\omega t) \leqslant D N(t) \quad \text { for } \quad t \text { sufficiently large, } \\
N(t)=N\left(\frac{t}{n} n\right) \leqslant D N(n) \quad \text { for } n \text { sufficiently large, } n \leqslant t \leqslant n+1,
\end{gathered}
$$

are satisfied, we can choose $r$ so great that for $N(t) \geqslant r, M(t) \geqslant r$

$$
D N(t) \geqslant M(t) \geqslant \frac{\varepsilon_{m}}{D} N(t) \text {. }
$$

The functions $N, M$ satisfy the condition (o), whence for $N(t)<r$ or $M(t)<r$ we have $M(t) \leqslant K$ or $N(t) \leqslant K, K$ being a constant, which gives $N \sim M$.

Stndia Mathematica T. XVII 
2.7. THEOREM 4. A. Suppose that $N$ is not bounded. Then $X^{N}$ forms a linear space if and only if:

(a) $N$ satisfies the condition (o);

(b) there are constants $C>0, r>0$ such that $N(t+s) \leqslant C(N(t)+N(s))$ for $|t|+|s|>r$

(c) for each $\varrho>0$ there exist constants $D>0, r_{0}>0$ such that $N(\omega t) \leqslant D N(t)$ for $|\omega|<\varrho,|t|>r_{0}$.

B. If $N$ is not bounded and $X^{N}$ is a linear space, we may replace $N$ by a continuous even function $M$ increasing for $t \geqslant 0$, satisfying the condition (*) and equivalent to $N$, i. e. such that $X^{M}=X^{N}$.

Ad A. Necessity. (a) follows by 2.2 (b) and (2.5); (b), (c) follow from 2.3, 2.4, and (a).

Sufficiency. The set $X^{N}$ is non-empty, e. $g . x(t)=0$ for every $t$ beongs to $X^{N}$, it is sufficient to apply the condition $(0)$ and 2.3 .

Ad B. Since $N$ must satisfy the condition (o), we may apply 2.6 .

2.71. If $N$ is non-decreasing for $t \geqslant 0, N(t)=N(-t)$ for all $t$, and $N$ satisfies the condition (o), then the conditions $2.7(\mathrm{~b}),(\mathrm{c})$ are equivalent to the following one:

$$
N(2 t) \leqslant K N(t)
$$

for sufficiently large $|t|, K$ being a constant.

The proof is analogous to that in 1.62 .

2.8. Let $X^{N}$ be a linear space; as in 1.8 , we introduce the notion of the $Q$-convergence. The sequence $x_{n} \in X$ is called $\varrho$-convergent to the element $x_{0} \in \bar{X}^{N}$ if $\varrho\left(x_{n}-x_{0}\right) \rightarrow 0$ as $n \rightarrow \infty$; we shall write this $: x_{n} \stackrel{\varrho}{\rightarrow} x_{0}$ as $n \rightarrow \infty$.

2.81. Let $X^{N}$ be a linear space and let $N$ not vanish identically. Then a necessary condition that the product $\omega x$ be continuous with respect to the o-convergence is that the condition $(\dot{*})$ be satisfied.

Under the supplementary hypothesis that $N$ satisfies the condition (o), (*) is a sufficient condition for the continuity of $\omega x$.

Necessity. Let $t_{n} \rightarrow 0$ as $n \rightarrow \infty, x_{n}(s)=1$ for $s \epsilon(0,1), n=0,1,2, .$. obviously $x_{n} \stackrel{\varrho}{\rightarrow} x_{0}$ as $n \rightarrow \infty$, whence $t_{n}, x_{n} \stackrel{\varrho}{\rightarrow} 0$, i. e. $\varrho\left(t_{n}, x_{n}\right)=\varrho\left(t_{n}, x_{n}\right)=$ $=N\left(t_{n}\right) \rightarrow 0$ as $n \rightarrow \infty$. Let $N\left(t_{n}\right) \rightarrow 0$ as $n \rightarrow \infty$; we may suppose that $t_{n} \rightarrow t_{0}$ or $\left|t_{n}\right| \rightarrow \infty$. Let us suppose also, for example, that $N(1) \neq 0$. Assuming $t_{0} \neq 0$ we may suppose that all $t_{n} \neq 0$. Setting $x_{n}(s)=t_{n}$ for $s \in(0,1) n=1,2, \ldots$ we get $x_{n} / t_{n} \stackrel{\varrho}{\rightarrow} 0$ as $n \rightarrow \infty$, whence $\varrho\left(x_{n} / t_{n}\right)=$ $=N(1) \rightarrow 0$ which is contradictory. Similarly we are led to a contradiction in the case $\left|t_{n}\right| \rightarrow \infty$; thus $t_{n} \rightarrow 0$. We omit here the proof of sufficiency, for it follows immediately from 2.84 .
Theorem 2.81 justifies the necessity of supposing the condition (*) when introducing in $X^{N}$ a norm such that the convergence with respect to the norm is equivalent to the $\varrho$-convergence (this equivalence is understood in the same sense as in 1.8).

2.82. Let $N$ satisfy the conditions (*), (0), and let $X^{N}$ be a linear space. Under these hypotheses the statements 1.81 (a), (b) are satisfied.

Let us observe at first that $N(0)=0$ and that $N$ is continuous at 0 . It follows by $2.7, \mathrm{~A}$, that for $x \in \bar{X}^{N}$ the inequality

$$
\int_{E} N(\omega x(t)) d t \leqslant D \int_{E} N(x(t)) d t \quad \text { when } \quad|\omega|<\varrho
$$

is satisfied where $E=\left\{t:|x(t)|>r_{0}, t \epsilon(0,1)\right\}, D, r_{0}, \varrho$ are constants as in $2.7, \mathrm{~A}$. Let $x_{n} \stackrel{\varrho}{\rightarrow} 0$; then $N\left(x_{n}(t)\right)$ converges in measure to 0 ; thus it follows from $(*)$ that $x_{n}(t)$ tends in measure to 0 . Let $\varphi_{n}$ denote the characteristic function of the set $E_{n}=\left\{t:\left|x_{n}(t)\right|>r_{0}, t \epsilon(0,1)\right\}$. As $\left(1-\varphi_{n}(t)\right) x_{n}(t)$ tends in measure to 0 and $\left|\left(1-\varphi_{n}(t)\right) x_{n}(t)\right| \leqslant r_{0}$ for $n=1,2, \ldots$, we have $\int_{0}^{1} N\left(\omega_{n}\left(1-\varphi_{n}(t)\right) x_{n}(t)\right) d t \rightarrow 0$ as $n \rightarrow \infty\left|\omega_{n}\right|<\varrho$. By $(+)$

$$
\int_{0}^{1} N\left(\varphi_{n}(t) \omega_{n} x_{n}(t)\right) d t \leqslant D \int_{0}^{1} N\left(\varphi_{n}(t) x_{n}(t)\right) d t \leqslant D \varrho\left(x_{n}\right)
$$

as $\left|\omega_{n}\right|<\varrho$ for $n=1,2, \ldots$, whence

$\varrho\left(\omega_{n} x_{n}\right)=\int_{0}^{1} N\left(\left(1-\varphi_{n}(t)\right) \omega_{n} x_{n}(t)\right) d t+\int_{0}^{1} N\left(\varphi_{n}(t) \omega_{n} x_{n}(t)\right) d t \rightarrow 0 \quad$ as $\quad n \rightarrow \infty$.

The proof of 1.81, (a) is analogous.

2.83. Let $N$ and $M$ satisfy the condition (*) and let $N \sim M$, i.e. $X^{N}=X^{M}$. Then $\varrho_{N}\left(x_{n}\right) \rightarrow 0$ as $n \rightarrow \infty$ impties $\varrho_{M}\left(x_{n}\right) \rightarrow 0$ as $n \rightarrow \infty$ and conversely.

It is sufficient to prove that $\varrho_{N}\left(x_{n}\right) \rightarrow 0, x_{n} \epsilon X^{N}$ implies $\varrho_{M}\left(x_{n}\right) \rightarrow 0$. According to $2.1(+)$ we have $M(t) \leqslant B N(t)$ for $N(t) \geqslant r$ and $M(t) \leqslant K$ for $N(t)<r$. Let $E_{n}=\left\{t: N\left(x_{n}(t)\right) \geqslant r, t \epsilon(0,1)\right\}$ for $n=1,2, \ldots$ and let $\varphi_{n}$ denote the characteristic function of the set $E_{n}$. We have

$$
\int_{0}^{1} M\left(\left(x_{n}(t)\right) \varphi_{n}(t) d t \leqslant B \int_{0}^{1} N\left(x_{n}(t)\right) \varphi_{n}(t) d t \leqslant B \varrho_{N}\left(x_{n}\right) \rightarrow 0 .\right.
$$

Now $M\left(x_{n}(t)\right)\left(1-\varphi_{n}(t)\right) \leqslant K, M\left(x_{n}(t)\right)$ tends in measure to 0 in $(0,1)$, for $N\left(x_{n}(t)\right)$ tends in measure to 0 and the condition (*) is satisfied. 
Thus

whence

$$
\int_{0}^{1} M\left(x_{n}(t)\right)\left(1-\varphi_{n}(t)\right) d t \rightarrow 0 \text { as } \quad n \rightarrow \infty
$$

$$
\varrho_{M}\left(x_{n}\right)=\int_{0}^{1} M\left(x_{n}(t)\right) d t \rightarrow 0 \quad \text { as } \quad n \rightarrow \infty .
$$

2.84. Let $N$ satisfy the conditions (*), (o), let $N$ be non-decreasing for $t \geqslant 0, N(t)=N(-t)$ for all $t$, and let $X^{N}$ be a linear space. One can define in $X^{N}$ an $F$-norm such that the convergence with respect to the norm is equivalent to the $\varrho$-convergence. With this norm, $X^{N}$ is an F'-space.

The set of the $\varepsilon>0$ satisfying the inequality $1.82(+)$ is non-empty, as follows by 2.82 . Let us define the norm as in $1.82, i . e .\|x\|=\inf \varepsilon$, the infimum being taken over the set of those $\varepsilon>0$ which satisfy 1.82 (+). Arguing as in 1.82 and taking into account the lemmata 2.82 we can verify that || || satisfies the axioms of $F$-norm and that the convergence with respect to the norm is equivalent to the $\varrho$-convergence. Let us call attention here to the role of the condition (*), considering, for example, the axiom: $\|x\|=0$ if and only $x=0$. If $\|x\|=0$, then $\varrho(x)=0$, for the convergence with respect to the norm implies the $\varrho$-convergence, and since $(*)$ implies $N(t) \neq 0$ for $t \neq 0$ we have $x(t)=0$ alimost everywhere, i.e. $x=0$. Conversely, if $x(t)=0$ almost everywhere, then $N(|x(t)| / \varepsilon)=0$ almost everywhere, $\varrho(x / \varepsilon)=0$, i.e. $\|x\|=0$. To prove the axiom of completeness ${ }^{2}$ ) let $M$ be a continuous even function satisfying the condition (*), equivalent to $N$; such a function exists in virtue of 2.6. Let $\left\|x_{p}-x_{q}\right\| \rightarrow 0$ as $p, q \rightarrow \infty$; by $2.83 \varrho_{M}\left(x_{p}-x_{q}\right) \rightarrow 0$ as $p, q \rightarrow \infty$. Let us choose $\varepsilon_{n}>0$ so that $\sum_{n} \varepsilon_{n}\left[M\left(1 / n^{2}\right)\right]^{-1}<\infty$ and then an increasing sequence $p_{n}$ of indices such that $\varrho_{M}\left(x_{p_{n}}-x_{p_{n+r}}\right) \leqslant \varepsilon_{n}$ for $n=1,2, \ldots$ Let $\quad E_{n}=\left\{t:\left|x_{p_{n}}(t)-x_{p_{n+1}}(t)\right| \geqslant 1 / n^{2}, t \epsilon(0,1)\right\}, F_{n}=(0,1)-E_{n} \quad$ for $n=1,2, \ldots$ The series $\sum_{n=1}^{\infty}\left|E_{n}\right|$ converges, for $\varrho_{M}\left(x_{p_{n}}-x_{p_{n+1}}\right) \geqslant\left|\boldsymbol{E}_{n}\right| M\left(1 / n^{2}\right)$, therefore $\left|\lim _{n} E_{n}\right|=0, \frac{\lim _{n}}{n} F_{n} \mid=1$, whence it follows that the series $\sum_{n}\left|x_{p_{n}}(t)-x_{p_{n+1}}(t)\right|$ converges almost everywhere, i.e. $x_{p_{n}}(t) \rightarrow x_{0}(t)$ almost everywhere. Let $\varepsilon>0$ be fixed; for sufficiently large $q$ and almost all $n$ we have $\int_{0}^{1} M\left(x_{p_{n}}(t)-x_{q}(t)\right) d t \leqslant \varepsilon$, which implies together with the continuity of $M$ that

$$
\lim _{n}^{1} \int_{0}^{1} M\left(x_{p_{n}}(t)-x_{q}(t)\right) d t \geqslant \int_{0}^{1} M\left(x_{0}(t)-x_{q}(t)\right) d t,
$$

2) For the quoted proof of the completeness of $X^{M}$ compare [1]. i.e. $\varrho_{M}\left(x_{0} \div x_{q}\right) \leqslant \varepsilon$ for large $q$. It follows that $x_{0} \in X^{N}, \varrho_{N}\left(x_{n}-x_{0}\right) \rightarrow 0$, whence $\left\|x_{n}-x_{0}\right\| \rightarrow 0$ as $n \rightarrow \infty$.

Taking into account 2.6, 2.84, and 2.83 we deduce

THEOREM 5. Every linear space of functions $X^{N}$ forms an $F$-space if $N$ satisfies the conditions $(0)$ and $(*)$; the $F$-norm may be defined so that the o-convergence is equivalent to the convergence with respect to the norm.

2.9. If $X^{N}$ is a locally convex $F$-space, $N$ satisfies the conditions (o), (*) and norm-convergence implies $\varrho$-convergence, then $N$ is equivalent to continuous convex even function satisfying the conditions $(*),(0)$.

Suppose that $X^{N}$ is a locally convex $F$-space; by the theorem of Banach and theorem 5 the convergence with respect to the norm is equivalent to the $\varrho$-convergence; by $2.6,2.83$ we may suppose that $N$ is continuous and increasing for $t \geqslant 0, N(t)=N(-t)$ for all $t$. The local convexity of $X^{N}$ implies the existence of $\varepsilon>0$ such that from the inequality $\varrho\left(x_{k}\right)<\varepsilon$ for $k=1,2, \ldots, n$ there follows $\varrho\left(\left(x_{1}+x_{2}+\ldots+x_{n}\right) / n\right)<1$. For positive integers $q, p, n$ such that $p \leqslant n$ and real $t$ let us define the functions $x_{k}$ for $k=1,2, \ldots, n$ as follows:

$x_{k}(s)=\left\{\begin{array}{l}t \varepsilon_{r(k-1+i)} \quad \text { for } \quad i / n q<s<(i+1) / n q, \quad i=0,1,2, \ldots, n-1, \\ 0 \quad \text { elsewhere in }(0,1),\end{array}\right.$

where $\varepsilon_{m}=1$ for $m=0,1, \ldots, p-1, \varepsilon_{m}=0$ for $m \geqslant p$ and $r(m)$ denotes the residue of $m$ modulo $n$. Now

$$
\varrho\left(x_{k}\right)=\frac{1}{q} \frac{p}{n} N(t), \quad \varrho\left(\frac{x_{1}+x_{2}+\ldots+x_{n}}{n}\right)=\frac{1}{q} N\left(\frac{p}{n} t\right),
$$

whence

$$
\frac{1}{q} N\left(\frac{p}{n} t\right)<1 \quad \text { for } \quad \frac{1}{q} \frac{p}{n} N(t)<\varepsilon
$$

The continuity of $N, p, n$ being arbitrary, implies that $q^{-1} N(\omega t) \leqslant 1$ for $0 \leqslant \omega \leqslant 1$ if $q^{-1} \omega N(t) \leqslant \varepsilon$. If $\omega N(t) \geqslant \varepsilon$ and $\varepsilon q \leqslant \omega N(t) \leqslant \varepsilon(q+1)$ for positive integer $q$, then

$$
\frac{1}{q+1} \omega N(t) \leqslant \varepsilon
$$

whence

$$
\frac{1}{q+1} N(\omega t) \leqslant 1, \quad N(\omega t) \leqslant q+1=\frac{q+1}{\varepsilon q} \varepsilon q \leqslant \frac{2}{\varepsilon} \omega N(t) .
$$

Setting $C=2 / \varepsilon$ we have

$$
(+) \quad N(\omega t) \leqslant C \omega N(t) \text { for } \omega N(t) \geqslant \varepsilon \text {. }
$$


We shall prove that

$$
\lim _{t \rightarrow \infty} \frac{N(t)}{t}>0
$$

In the contrary case there exist $t_{n}$ such that $t_{n} \rightarrow \infty, N\left(t_{n}\right) / t_{n} \rightarrow 0$ as $n \rightarrow \infty$. Now $N\left(t_{n}\right) \rightarrow \infty$, since $N$ satisfies the condition (o) and we may suppose that $\omega_{n}=\varepsilon / N\left(t_{n}\right) \leqslant 1$. As $\omega_{n} N\left(t_{n}\right)=\varepsilon$ we infer from $(+)$ that

$$
N\left(\varepsilon \frac{t_{n}}{N\left(t_{n}\right)}\right) \leqslant \theta_{\varepsilon}
$$

which contradicts the condition (0), for $\varepsilon t_{n} / N\left(t_{n}\right) \rightarrow \infty$ as $n \rightarrow \infty$. Let us choose $\delta>0$ and $T>0$ so that

$$
N(t) / t \geqslant \delta \text { for } \quad t \geqslant T \text { and } \delta T \geqslant \varepsilon .
$$

Then for $\omega t \geqslant T, 0<\omega \leqslant 1, t>0$

$$
\omega N(t)=\omega t \frac{N(t)}{t} \geqslant T \delta \geqslant \varepsilon,
$$

for $\omega t \geqslant T$ implies $t \geqslant T$; therefore, by $(+)$,

$(++)$

$$
N(\omega t) \leqslant O \omega N(t) \quad \text { for } \quad \omega t \geqslant T, 0<\omega \leqslant 1 .
$$

Let us set

$$
P(t)= \begin{cases}\sup _{0<\omega \leqslant 1} \frac{N(\omega t)}{\omega} \quad \text { for } \quad t \geqslant T \\ N(t) & \text { for } \quad 0 \leqslant t<T .\end{cases}
$$

From $(++)$ and the continuity of $N$ it follows that $P(t)<\infty$ for every $t$. The following inequality is satisfied:

for

$$
P(\alpha t) \leqslant \alpha P(t) \quad \text { as } \quad 0<\alpha \leqslant 1, \alpha t \geqslant T
$$

$$
P(\alpha t)=\sup _{\substack{0<\omega \leqslant 1 \\ \omega \alpha t \geqslant T}} \frac{N(\omega \alpha t)}{\omega}=\operatorname{sip}_{\substack{\alpha<\omega \leqslant 1 \\ \omega \alpha t \gg T}} \frac{N(\omega a t)}{\omega \alpha} \leqslant \sup _{\substack{o<\omega \leqslant 1 \\ \omega t \geqslant T}} \frac{N(\omega t)}{\omega}=\alpha P(t) ;
$$

it follows that the function $P(t) / t$ is non-decreasing as $t \geqslant T$, since for $T \leqslant t^{\prime}<t^{\prime \prime}$

$$
\frac{P\left(t^{\prime}\right)}{\boldsymbol{t}^{\prime}}=\frac{P\left(\frac{t^{\prime}}{t^{\prime \prime}} t^{\prime \prime}\right)}{t^{\prime}} \leqslant \frac{\frac{t^{\prime}}{t^{\prime \prime}} P\left(t^{\prime \prime}\right)}{t^{\prime}}=\frac{P\left(t^{\prime \prime}\right)}{t^{\prime \prime}}, \quad \text { for } \quad \frac{t^{\prime}}{t^{\prime \prime}} t^{\prime \prime}=t^{\prime} \geqslant T .
$$

Let us now write

$$
\begin{gathered}
Q(t)= \begin{cases}P(t) / t & \text { for } \quad t \geqslant T, \\
t P(T) / T^{2} & \text { for } \quad 0 \leqslant t \leqslant T,\end{cases} \\
M(t)=\int_{0}^{t} Q(s) d s \quad \text { for } t \geqslant 0, \quad M(t)=M(-t) \text { for } \quad t \leqslant 0 .
\end{gathered}
$$

The function $M$ is convex and, as in 1.91, one can prove that $N \sim M$.

2.91. If $X^{N}$ is a linear space, $N$ satisfies the conditions $(0),(*)$ and $N$ is equivalent to a convex function, then $X^{N}$ is a B-space with norm-convergence equivalent to $\varrho$-convergence.

Let the function $M$ be convex and equivalent to $N$; this, together with (o), implies that $M$ is continuous. We may assume that $M$ is even; the condition (0) is satisfied by $M$, for it is satisfied by $N$. It is easily seen that modifying suitably $M$ in a neighbourhood of 0 we may obtain a convex function equivalent to $M$ satifying the condition (*); thus we can suppose that $M$ satisfies (*). Using 2.84 and arguing as in $1.91^{3}$ ) we can prove that the norm described in lemma 2.84 is equivalent to a $B$-norm.

2.92. As a corollary to $2.9,2.91$ and applying 2.71 we get

THeOREM 6. (a) Let $N$ satisfy the conditions (o) and (*). Then $X^{N}$ is a Banach space if and only if $N$ is equivalent to a continuous even convex function vanishing only at 0 and satifying the inequality $2.71(+)$.

(b) If $X^{N}$ is a $B_{0}$-space, then $X^{N}$ is a B-space.

Here norm-convergence is always to understand as equivalent to @-convergence.

\section{References}

[1] S. Kaczmarz et L. Nikliborc, Sur les suites des fonctions convergentes en moyenne, Fundamenta Mathem. 11 (1928), p. $151-168$.

2] W. A. J. Luxembourg, Banach function spaces, thesis, Delft 1955.

[3] W. Orlicz, Über eine gewisse Klasse von Räumen vom Typus B, Bull. Ac. Pol. (1932), p. $207-220$.

[4] - Über Räume $\left(L^{M}\right)$, Bull. Ac. Pol. (1936), p. 93.107. Ann. 69 (1910), p. $449-497$.

[6] - Les systèmes d'équations linéaires à une infinité d'inconnues, Collection Borel, Paris 1913 .

Reģu par la Rédaction le 21. 9. 1957

s) Compare the footnote on p. 105. 